\title{
Developing a framework for gathering and using service user experiences to improve integrated health and social care: the SUFFICE framework
}

Vicky Ward ${ }^{*}$, Lisa Pinkney and Gary Fry

\begin{abstract}
Background: More people than ever receive care and support from health and social care services. Initiatives to integrate the work of health and social care staff have increased rapidly across the UK but relatively little has been done to chart and improve their impact on service users. Our aim was to develop a framework for gathering and using service user feedback to improve integrated health and social care in one locality in the North of England.

Methods: We used published literature and interviews with health and social care managers to determine the expected service user experiences of local community-based integrated teams and the ways in which team members were expected to work together. We used the results to devise qualitative data collection and analysis tools for gathering and analyzing service user feedback. We used developmental evaluation and service improvement methodologies to devise a procedure for developing service improvement plans.

Findings: We identified six expected service user experiences of integrated care and 15 activities that health and social care teams were expected to undertake. We used these to develop logic models and tools for collecting and analysing service user experiences. These include a narrative interview schedule, a plan for analyzing data, and a method for synthesizing the results into a composite 'story'. We devised a structured service improvement procedure which involves teams of health and social care staff listening to a composite service user story, identifying how their actions as a team may have contributed to the story and developing a service improvement plan.

Conclusions: This framework aims to put service user experiences at the heart of efforts to improve integration. It has been developed in collaboration with National Health Service (NHS) and Social Care managers. We expect it to be useful for evaluating and improving integrated care initiatives elsewhere.
\end{abstract}

Keywords: Integrated care, Service user experience, Health care, Social care, Service improvement

\section{Background}

In the United Kingdom (UK) a rising population of older adults and a significant increase in the number of people with complex health needs means that more people than ever are receiving care and support from both health and social care services. Recent figures show that 15 million people have one or more long-term conditions and their

*Correspondence: v.l.ward@leeds.ac.uk

Leeds Institute of Health Sciences, University of Leeds, 101 Clarendon Road, Leeds LS2 9LJ, UK care accounts for $70 \%$ of the primary and acute care budget in England [1] whilst around 1.1 million people receive care at home, $80 \%$ of whom are state supported [2]. The coordination of care and support for these people has historically been poor resulting in people falling through gaps in service provision, being 'bounced around' different services and having to explain themselves and their needs multiple times [3]. Poor coordination also results in duplication between health and social care services and unnecessary hospital stays, further increasing the financial burden on the system [4]. 
As a result there is currently a significant policy drive towards integrating health and social care support [5] and improving the experiences of service users with complex needs [6].

A range of integrated care initiatives have been implemented across the UK in recent years. In 2009 the Department of Health launched a 2-year pilot programme to explore and evaluate different models of integrated care [7]. In 2013 this was followed by the naming of 14 'integration pioneers' across the UK where innovative ways of delivering coordinated care and bringing services closer together are being pursued [8]. These and other sites have used a range of approaches including multidisciplinary meetings [9], risk profiling and case management [10] and pooled service and commissioning budgets [11]. The majority of initiatives in the UK have tended to take place at a micro level where providers seek to deliver integrated care for individual service users through care co-ordination, care planning, use of technology and other approaches [12]. Such initiatives include setting up community-based co-located teams of nurses, social workers, occupational therapists and physiotherapists [13-15].

In an effort to learn lessons about how best to provide integrated health and social care and the impact of integrated working, recent initiatives have been the subject of a number of evaluations. Evaluative activities have largely focused on emergency hospital admissions and the barriers to integrated working from the perspective of health and social care staff $[10,16,17]$ and findings to date have not been especially positive. The national evaluation of the UK's integration pilot programme, for instance, showed no evidence that pilot sites were reducing the level of emergency hospital care [7], whilst a number of studies have highlighted a range of barriers to integrated working including professional/disciplinary mismatches, lack of clarity about the purpose of integration, lack of understanding and clarity about each other's roles and the use of rhetorical claims and ideals to quash real experiences [14].

There has been less emphasis on how integrated ways of working are impacting on service users and how they can be designed to provide service users with a better experience of care. Evidence to date suggests that there is frequently a mismatch between the aims and impact of integration on service user experiences [7, 18], but there is general agreement that service user experiences of integrated care are not well defined or appropriately captured, especially by those organisations who are actively developing and implementing integrated care initiatives $[3,19]$. Indeed, previous evaluative efforts have been criticized by service users and other stakeholders for focusing on descriptions of what integrated care should look like and what organisations should do, rather than on the voices and experiences of service users [19].

By failing to capture how service users are experiencing efforts to improve integration and coordination, organisations are also missing a potentially powerful catalyst for improving integrated working. During recent consultations, service users and other stakeholders across the UK were clear that efforts to evaluate service user experiences of integrated care should be used for driving improvement and encouraging better communication and joint working between health and social care professionals [19]. This is supported by evidence on interprofessional teamworking, which shows that having a shared sense of purpose focused around improving outcomes and experiences for service users is a key enabler of interprofessional teamwork [20, 21]. As Cameron et al. [22] found, expressing the benefits of joint working in terms of client outcomes/experiences helps staff to recognise that they need to work together to achieve these.

This paper focuses on the development of a framework for gathering and using service user feedback to improve integrated working between health and social care staff. Named SUFFICE (Service User Feedback Framework for Improving integrated CarE), the framework was developed at the request of health and social care organisations in a city in the North of England, who recognized their need to understand how service users were experiencing their local efforts to improve coordination and integrated working across the city and respond to those experiences. In the remainder of this paper we detail the methods used to develop the framework and describe the contents of the framework.

\section{Methods \\ Developing the framework \\ Setting}

In early 2012 the local vision for integration in our study site proposed that General Practitioners (GPs), health workers and social care staff would work side-by-side in close knit teams, identifying levels of risk, sharing information and taking a joint approach to supporting older people and those with long-term conditions. One of the first strands of work was the co-location of health and social care staff into 12 'integrated neighbourhood teams', each of which served a specific geographical area. Teams were set up over a 12 month period between February 2012 and February 2013. These teams were chosen as the focus for our framework because they were seen as a particularly important element of the local integration effort, were a relatively well-defined intervention which could be distinguished from the usual ways of working, and offered a forum to engage staff in improving integrated 
working by reflecting on service user feedback. We were asked to design the framework in early 2013.

\section{Design}

The overall aim of our work was to design a practical and user-friendly framework which could be used by those commissioning and delivering integrated health and social care services to understand and respond better to the experiences of service users and their carers. The expectation of those who were setting up the teams was that they would develop and evolve over time. This led us to select developmental evaluation approaches as the basis for our work since these aim to support the development and adaptation of interventions in dynamic environments by focusing on the co-creation of useful and practical evaluation tools [23]. Our approach meant that we worked closely with staff from relevant health, social care and service user organisations throughout the project. This included establishing a project management group which included representatives from the research team and from local health, social care and voluntary sector organisations. This provided a forum for reporting progress back to the organisations who had requested the framework, and ensured that the framework itself would be fit-for-purpose.

Phase 1: understanding and uncovering expectations about integrated care experiences and practices We divided the project into several phases. In the first phase we focused on understanding service user experiences of and expectations about integrated care and what effective interprofessional teamworking looks like. We operationalised these as two inter-related questions to help us better communicate our focus to our local partners: 'What experiences can we expect integrated working to deliver for service users?' and 'How should we expect integrated neighbourhood teams to work together?'

In line with our developmental evaluation approach, our overall aims in this phase of the work were to determine the type of service user feedback that would be useful for developing integrated working, the type of activities that integrated teams could be encouraged to engage in, and develop a shared understanding (with our local partners) of how integrated neighbourhood teams could improve service user experiences of care.

We undertook three activities to enable us to answer these questions. First, discussions with our local partners led us to identify two initiatives (one local and one national) to develop outcome frameworks for integrated care. The local framework was based on interviews with service users and staff about what they wanted and expected from local integration efforts [24]. The national initiative was undertaken by National Voices (a coalition of health and social care charities in England) and involved interviewing and working with service users to produce narratives of person-centred, coordinated care [25]. We used both of these sources to develop initial lists of service user experiences and teamworking attributes and practices which could be associated with integrated care.

Second, we conducted two scoping reviews. These were specifically requested by our local partners in order to help them understand the current knowledge base on integrated working. Our first review focused on published accounts of service user's experiences of integrated care. Our second review focused on published accounts of how staff from different organisational and professional backgrounds work together and the markers of effective teamworking. Our search strategies and results can be seen in Table 1.

Two team members (VW and LP) read the papers which met our inclusion criteria in full and worked together to extract and summarise service user's experiences of integrated care and the features of integrated teamworking and develop these into two sets of short statements. We also used the results of the reviews to produce short documents for our project partners which summarised the knowledge base in relation to these aspects of integrated care.

Our third activity involved conducting interviews with 15 local commissioning and service provider managers, including members of the local Integrated Health and Social Care Board. We decided not to interview service users since we were confident that the work undertaken locally and nationally adequately captured service user expectations about integrated care and we did not wish to overburden local service users. Written consent for the interviews was given by all participants. We asked interviewees what they hoped that service users would say after receiving care from an Integrated Neighbourhood Team and what the teams would need to do to achieve those positive experiences. Interviews were recorded and transcribed in full. Two team members (VW and LP) categorized interview material into 'service user experiences' and 'team activities', summarized it (by paraphrasing interviewees key points) using the framework function in NVivo 9 (QSR International) and used the resulting framework matrix to identify the service user experiences and team activities discussed by our interviewees.

Two team members (VW and LP) compared the service user experiences which we had identified from the local and national outcome frameworks, scoping reviews and interviews. We discussed, grouped and summarised these to develop a final list of six service user experiences associated with integrated care. We used the same approach to develop a list of 15 attributes and activities associated with integrated teamworking. 
Table 1 Scoping review search criteria and results

\begin{tabular}{|c|c|c|}
\hline & Service user experiences & Interprofessional teamworking \\
\hline Eligibility criteria & $\begin{array}{l}\text { Published papers describing service user experiences of } \\
\text { integration in health and social care } \\
2008-2013 \\
\text { English language }\end{array}$ & $\begin{array}{l}\text { Published papers describing integrated teamworking between } \\
\text { health and/or social care professionals in community settings } \\
\text { 2003-2013 } \\
\text { English language }\end{array}$ \\
\hline Databases & $\begin{array}{l}\text { MEDLINE } \\
\text { EMBASE } \\
\text { HMIC } \\
\text { Social care online } \\
\text { ASSIA }\end{array}$ & $\begin{array}{l}\text { MEDLINE } \\
\text { EMBASE } \\
\text { HMIC } \\
\text { PSYCInfo } \\
\text { ASSIA } \\
\text { Social services abstracts } \\
\text { PAIS international } \\
\text { Scopus (health sciences, social science) }\end{array}$ \\
\hline Hand searches & $\begin{array}{l}\text { International journal of integrated care } \\
\text { Journal of integrated care }\end{array}$ & $\begin{array}{l}\text { International journal of integrated care } \\
\text { Journal of integrated care }\end{array}$ \\
\hline Search terms & Social care AND health AND integrated & $\begin{array}{l}\text { Health OR social care AND [integrat* AND team* AND multi*] } \\
\text { AND [community OR primary care] AND [case study OR evaluat* } \\
\text { OR interview OR ethnograph* OR focus group OR survey OR } \\
\text { questionnaire OR observat*] }\end{array}$ \\
\hline $\begin{array}{l}\text { Inclusion/exclusion } \\
\text { criteria }\end{array}$ & $\begin{array}{l}\text { Include: papers which provide empirical evidence of service } \\
\text { user experiences } \\
\text { Exclude: papers which only focus on staff perceptions of ser- } \\
\text { vice user experiences, service or system-level outcomes, } \\
\text { descriptions of integration schemes }\end{array}$ & $\begin{array}{l}\text { Include: papers which provide empirical evidence of how mul- } \\
\text { tiprofessional teams work together to provide care to service } \\
\text { users in community settings } \\
\text { Exclude: theoretical papers and papers which focus on single } \\
\text { professional groups }\end{array}$ \\
\hline $\begin{array}{l}\text { No. of returned } \\
\text { papers }\end{array}$ & 413 & 904 \\
\hline $\begin{array}{l}\text { No. of papers } \\
\text { meeting inclusion } \\
\text { criteria }\end{array}$ & 47 & 63 \\
\hline
\end{tabular}

Phase 2: developing a logic model The second phase of the project involved linking the service user experiences and team activities and developing a series of 'logic models' [23]. This type of model is widely used in program evaluations as a way of helping program developers to clarify their goals and planned activities and for visualizing the linkages between them. Since our overall aim was to develop a framework which would enable staff working in the neighbourhood teams to explicitly understand the links between service user experiences and their activities and working practices (and make any necessary changes), we judged that logic models could be a useful part of this framework. The logic models were also designed to act as a tentative roadmap for service providers and commissioners by showing how the Integrated Neighbourhood Teams were designed to work and where any adjustments needed to be made. Recognising that it was important to co-produce the logic models with staff who had a more detailed view of the Integrated Neighbourhood Teams, we held a $2 \mathrm{~h}$ workshop with local commissioning and operational managers and a service user involvement specialist. During the workshop we worked with these managers to group together the team activities which were most likely to influence each of the six service user experiences and to arrange them into a logical chain of events. We used the outputs from this meeting to produce draft versions of the models which were circulated to our project collaborators for further comments and adaptation.

Phase 3: devising tools for gathering experiences of integrated care The third phase of the project involved devising tools for collecting and analysing service user experiences. Some of our project partners originally envisioned that survey tools could be used to collect service user experiences but after a number of group discussions about the difficulties of administering and collecting surveys from the frail older adults who were most likely to be receiving care via an Integrated Neighbourhood Team [26] and the limitations of survey data for understanding individual experiences, we decided to focus on capturing rich descriptions of people's experiences in their own words using a qualitative approach [27].

We recognised that qualitative approaches have the potential to generate a vast amount of data which can be difficult and time-consuming to analyse. This had the potential to clash with our overall aim of developing a practical framework which could be used by staff with relatively little research experience. One team member (GF) therefore reviewed a range of academic and grey literature about the collection and analysis of qualitative 
data by lay researchers and service users [28-30] and identified a number of key principles. These included dividing the data collection and analysis process into a series of clear and well-defined tasks, developing a topic guide flexible enough to meet the needs of interviewees, using a simplified coding framework and developing creative ways of presenting findings that may help to overcome resistance from staff members. We drew on these principles and our own experience and knowledge of qualitative analysis techniques to devise a comprehensive series of data collection and analysis tasks, a procedure for working with interview data direct from audio recordings [31], a simple analysis codebook [32] and a procedure for comparing and summarizing service user experiences into a single narrative story. As with the previous phases, we worked closely with our local partners in developing these materials and approaches.

Phase 4: devising tools for developing service improvement plans The final phase of the project focused on devising a procedure to enable the Integrated Neighbourhood Teams to reflect on and develop their teamworking practices by reflecting on the experiences of their service users. To develop the procedure we drew on two main resources. In line with our overall emphasis for the project, the first was developmental evaluation methodology [23], which focuses on the innovative use of formative feedback (and other resources) to improve and develop an ongoing intervention (such as integrated neighbourhood teams). The second was NHS service improvement resources [33]. As well as focusing on facilitating collaborative problem-solving, understanding and mapping current practices and planning activities to improve those practices, many of these have also been developed and tested in NHS contexts and were familiar to most of our partner organisations. In collaboration with our project partners, we used these sources to devise a structured protocol which would enable teams to reflect on the experiences of their service users, identify how their work impacts on those experiences and develop a clear service improvement plan to address any identified issues.

Between January and April 2014 the SUFFICE framework was implemented with three Integrated Neighbourhood Teams by our partner organisations. They took full responsibility for implementing the framework (including collecting and analysing the service user feedback data and arranging service improvement planning meetings). In the interests of data protection and brevity, we cannot present the results of the work conducted by our partner organisations or comment on the implementation of the SUFFICE framework.

In the following section we describe the contents of the SUFFICE framework in more detail.

\section{Results}

\section{Suffice framework materials}

In this section we describe the SUFFICE framework materials in the order in which they were developed. We begin by presenting the outputs of phases 1 and 2-our models of the expected linkages between integrated care experiences and practices. We then present the tools for gathering integrated care experiences (phase 3) before detailing the tools for developing service improvement plans (phase 4). In practice, the SUFFICE materials are designed to be used in a different order to that in which they were developed, and so we round the section off with a flow diagram showing how the SUFFICE framework is designed to be operationalised (Fig. 2).

\section{Logic models: linking integrated care experiences and practices}

From our literature review, interviews and review of previous outcome frameworks we identified six service user experiences which are expected to arise from an increase in integrated working. To make them readily accessible to a range of audiences, and in line with the influential work undertaken by National Voices to produce coordinated care narratives [25] and advice from our project partners, we expressed these as a series of 'I' statements. Four statements are related to a nominal service users' journey, from assessment through to receipt of care from a new service, whilst the other two relate to care processes in general.

1. When my needs are being assessed and my package of care is being put together (or altered) I do not keep having to say the same thing to lots of different people.

2. When the care and support I need has been agreed, I receive it in an efficient and timely manner-things happen when they are supposed to.

3. When my needs change or things go wrong I know who to contact/who to go to/what to do-I am not bounced around the system.

4. When I need care and support from a new service (e.g. hospital), they already know what my needs are and who else is involved in providing me with care and support.

5. My package of care and support is focused on me and my needs - my opinion is listened to and respected.

6. I know about the range of formal and informal support that is available to me.

From our literature review and interviews we identified 15 markers of and activities associated with integrated teamworking. 
1. Teams have networks across a number of agencies and work closely with those agencies.

2. Team members are able to work across geographical boundaries.

3. Team members understand one another's background and culture.

4. Teams offer a full range of support. They are able to access and signpost to specialist and community support.

5. Teams have a shared identity in which all team members are engaged.

6. Teams clearly identify one case manager for each service user.

7. Teams identify who is best placed to do an assessment or care planning.

8. The team carries out one joint comprehensive assessment.

9. The team shares information with other agencies about individual service users.

10. Staff are able to blur boundaries and share work with each other.

11. Teams are comfortable working closely together.

12. Teams have regular meetings to discuss shared cases.

13. Teams respond quickly to service user need.
14. Team members are focused on their service user's needs.

15. Teams are able to make efficient use of time.

During the workshop and subsequent discussions with local managers, these sets of expectations were adapted and linked together to produce a series of six 'logic models' (one for each expected service user experience). Each model includes team activities (shown in rounded boxes), team-level outcomes (shown in brackets) and the expected service user experience (shown in a coloured box on the right of the diagram). Dotted arrows link many of the team activities and outcomes to demonstrate the expected logical sequence of events. An example of a logic model can be seen in Fig. 1 below. All of the logic models can be found in Additional file 1: Appendix S1.

The main purpose of the logic models is to help facilitate structured reflection and the development of service improvement plans by team members. This led us to include several additional elements in each logic model. First, to facilitate structured reflection on the part of the teams, each activity and outcome is expressed from the perspective of the team ('we' statements). Second, each activity and outcome also includes a box for team

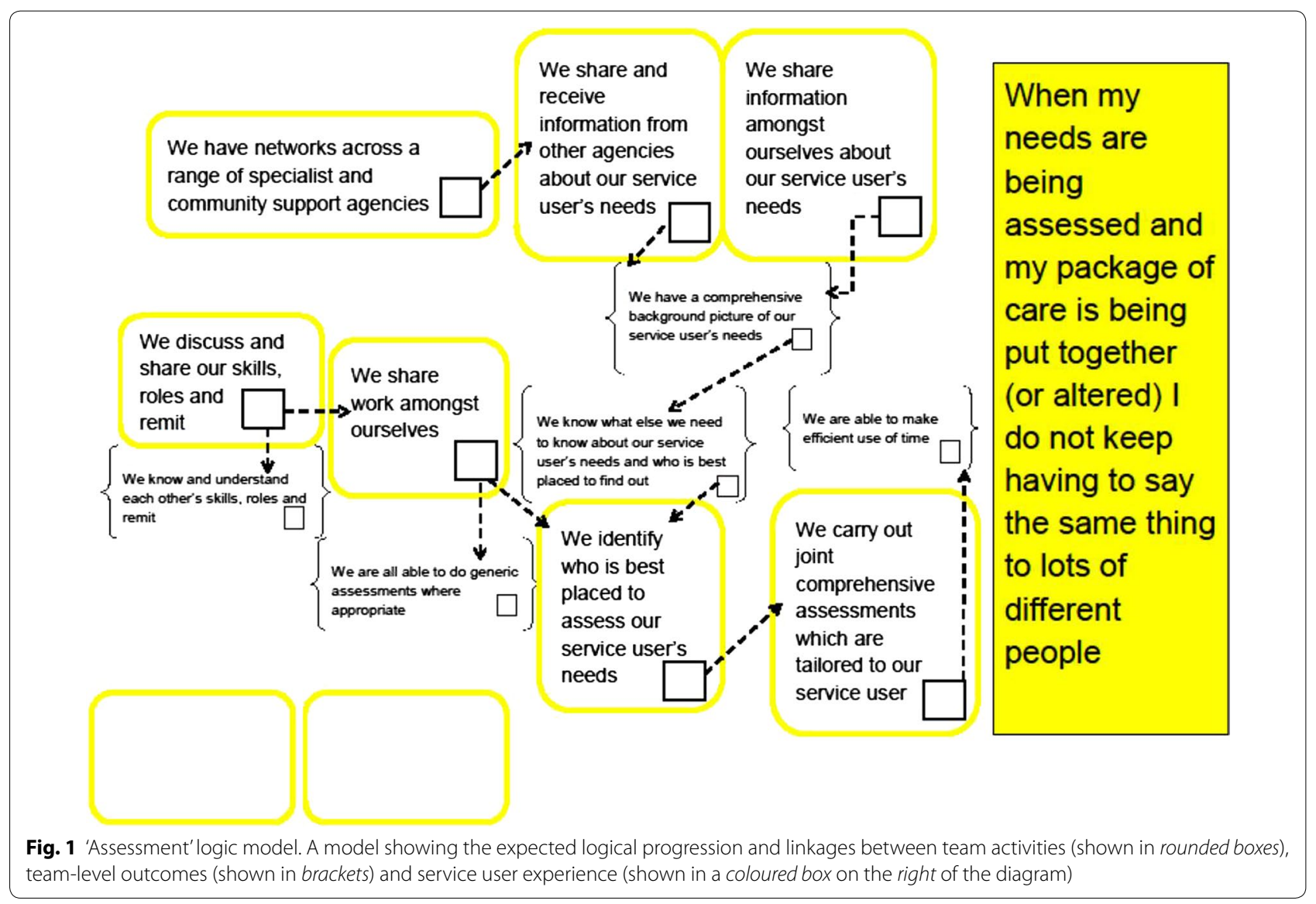


Table 2 overview of the service user experiences interview schedule with examples

\begin{tabular}{|c|c|c|}
\hline $\begin{array}{l}\text { Interview sec- } \\
\text { tion }\end{array}$ & Description & Interview schedule example \\
\hline $\begin{array}{l}\text { Introduction/ } \\
\text { basic informa- } \\
\text { tion }\end{array}$ & $\begin{array}{l}\text { This section focuses on gathering basic details } \\
\text { about the service user and their current } \\
\text { situation. It is also designed to help the inter- } \\
\text { viewer and interviewee develop a rapport }\end{array}$ & $\begin{array}{l}\text { Please tell me a little about yourself/the person you care for } \\
\text { Do you/they have any particular health conditions? } \\
\text { How old are you/they? }\end{array}$ \\
\hline Timeline & $\begin{array}{l}\text { This section involves using a simple timeline } \\
\text { to record significant events and experiences } \\
\text { over the past } 6-12 \text { months. It is designed to } \\
\text { be a visual tool to help focus the interview } \\
\text { and identify key episodes to explore in more } \\
\text { detail }\end{array}$ & $\begin{array}{l}\text { Instruction to interviewer: Use the timeline tool to identify key events and to gen- } \\
\text { erate discussion about the interviewee's experience of health and social care } \\
\text { Question: Could you tell me about the care and support you/the person you are } \\
\text { caring for has received over the last } 6-12 \text { months? }\end{array}$ \\
\hline $\begin{array}{l}\text { Key events/epi- } \\
\text { sodes }\end{array}$ & $\begin{array}{l}\text { This section involves focusing in more detail } \\
\text { on the key events experienced by the service } \\
\text { user. It includes a series of prompts to help } \\
\text { explore the different types of event that a } \\
\text { service user might have experienced (assess- } \\
\text { ment, receiving care and support, changing } \\
\text { needs/crisis, accessing new services) }\end{array}$ & $\begin{array}{l}\text { Instruction to interviewer: Using the timeline as a guide, focus on key events where } \\
\text { things seemed to have gone well, along with those where things seemed to go } \\
\text { wrong. Ask the interviewee to explore what happened and why they think things } \\
\text { went well/badly. } \\
\text { Assessment prompts: How much time did the person assessing/planning your } \\
\text { care spend with you? Did it feel like enough time? } \\
\text { How many people did you see during the assessment process? } \\
\text { Who were they? } \\
\text { What did they do/ask you? } \\
\text { Where did you see them? } \\
\text { Did you feel listened to? }\end{array}$ \\
\hline
\end{tabular}

members to indicate whether they feel that each of them occurs in their team. Finally, empty boxes are included so that they can also suggest other important activities not represented in the model.

\section{Tools for gathering and analysing service user experiences}

Since we were interested in gathering the experiences of service users in their (or their carers') own words, the data collection tools are not driven by the six expected experiences represented in the logic models. Instead, we devised a semi-structured interview schedule divided into three sections, as shown in Table 2. The full interview schedule is available in Additional file 2: Appendix S2.

The six expected service user experiences were used to drive the analysis plan, which is divided into three phases.

1. Familiarisation and identifying relevant material. This phase involves listening to an audio recording of each interview and noting the content using a timed grid. The aim of this phase is to identify the points at which various topics are discussed and start to identify material which relates to the six expected service user experiences.
2. Coding and summarizing. This phase involves listening in detail to key points of analytical interest (i.e. material which relates to the six service user experiences) and producing detailed summaries of this material guided by an analysis codebook. An extract from the codebook is shown in Table 3. The full analysis codebook can be found in Additional file 3: Appendix S3.

3. Comparing and synthesizing. This phase involves transferring the summaries produced for each interview into a simple table to enable the comparison of experiences between interviewees. The aim of this phase is to summarise the key points of similarity and difference in relation to each expected experience across several interviews. An example can be seen in Table 4.

To enable these key points to be communicated to a range of audiences and used as the basis for developing service improvement plans, we devised a mechanism for constructing composite stories based on the experiences of several service users. Stories are increasingly being used as a way of communicating service user experiences and have been shown to be a powerful catalyst for service redesign and change by inspiring 
Table 3 Extract from the service user experiences analysis codebook

Theme 1: When my needs are being assessed and my package of care is being put together (or altered) I do not have to keep saying the same thing to a lot of different people

This section focuses on assessments and how the plan of care was produced if at all. Focus on parts of the interview where the interviewee talks about:

Someone talking to them about their needs

Having their needs assessed

How assessments were carried out

How many/which people were involved in assessing their needs

Having to repeat their needs to different people

How their care was planned

Who was involved in planning their care

Notes/summary

Interesting quotes

understanding and empathy, and encouraging service providers to listen, learn, and act upon what they are told [34, 35]. The mechanism we developed focuses on producing separate stories to illustrate each of the six service user experiences. Our materials include a simple template and style guide which make it clear that whilst the decision about how to weave together the key points rests with the analyst (which may include embellishing contextual details and circumstances), the focus of the story should remain on the key points which emerged from the analysis.

\section{Tools for structured reflection and planning}

We developed a structured protocol to guide teams through the process of developing a service improvement plan over the course of one or two meetings. The protocol includes four separate stages, drawing on the composite stories of service user experiences and the logic models, which culminate in an agreed service improvement action plan. The protocol also includes instructions about selecting a facilitator and scribe for the group discussion and someone to take responsibility for coordinating the implementation of the agreed service improvement plan. An overview of the four stages and example text from the protocol is shown in Table 5 below. The complete service improvement protocol can be seen in Additional file 4: Appendix S4.

The complete SUFFICE framework is shown in Fig. 2 below, which demonstrates how the materials we have described fit together in practice.

\section{Conclusions}

In this paper, we have presented an innovative framework for gathering and using service user feedback to inform ongoing service improvement in integrated care.

The SUFFICE framework is important in two respects. First, gathering and evaluating service user experiences are neglected aspects of the integrated care landscape, with the focus tending to remain on idealised descriptions of integrated care from an organisational perspective. The SUFFICE framework aims to rebalance this by providing a mechanism for producing realistic composite stories which represent the voices and experiences of those receiving integrated care services. Second, enabling teams of staff to develop a shared sense of purpose focused on the needs of their service users is a crucial aspect of improving integrated working between health and social care staff. The SUFFICE framework provides a mechanism for teams to make plans for improving integrated working which have service user experiences at their heart.

Table 4 Table for comparing and synthesizing service user experiences

\begin{tabular}{llll}
\hline & Interview $\mathbf{1}$ & Interview $\mathbf{2}$ & Summary of key points \\
\hline Section 3 crisis & KW always knew which provider the care & At night, the only emergency number & Both KW and PB experienced difficul- \\
workers came from, but not who to & PB had was for a GP, but would have & ties contacting staff in the event of an \\
contact in the event of a problem. There & $\begin{array}{l}\text { preferred a number for a nurse; She won- } \\
\text { emergency }\end{array}$ & $\begin{array}{l}\text { ders whether other people, less willing } \\
\text { to bother staff, might be less likely to get } \\
\end{array}$ \\
& appropriate support
\end{tabular}


Table 5 An overview of the service improvement protocol

\begin{tabular}{|c|c|c|}
\hline Stage & Description & Improvement protocol example \\
\hline $\begin{array}{l}\text { Stage 1: storytelling and } \\
\text { initial reactions }\end{array}$ & $\begin{array}{l}\text { The team is told a composite story illustrating service user experi- } \\
\text { ences in relation to one of the six expected experiences and } \\
\text { has an opportunity to give some initial reactions. The aim is to } \\
\text { provide everyone with an opportunity to air and 'park' any initial } \\
\text { thoughts, reactions, questions or concerns so that they do not } \\
\text { become a distraction during the following stages }\end{array}$ & $\begin{array}{l}\text { After listening to the story, please invite team members } \\
\text { to give one initial thought/impression and make a note } \\
\text { of them here }\end{array}$ \\
\hline $\begin{array}{l}\text { Stage } 3 \text { : selecting an } \\
\text { area for improvement }\end{array}$ & $\begin{array}{l}\text { Team members select where to focus their service improvement } \\
\text { efforts by discussing the results of the previous stage using } \\
\text { a series of prompts. At the end of this stage, teams use the } \\
\text { protocol to record their decisions about the activities that they } \\
\text { have decided to focus on. If the process is to be carried over to } \\
\text { a second meeting, teams also record the person who will lead/ } \\
\text { coordinate those efforts and the date by which they will have } \\
\text { devised a concrete service improvement plan }\end{array}$ & $\begin{array}{l}\text { Which activities are likely to have had the most influence } \\
\text { on the service user story? } \\
\text { What should we keep doing/do more of to deliver posi- } \\
\text { tive experiences for our service users? } \\
\text { What do we need to start/fix to deliver better experi- } \\
\text { ences for our service users? }\end{array}$ \\
\hline $\begin{array}{l}\text { Stage 4: developing a } \\
\text { service improvement } \\
\text { plan }\end{array}$ & $\begin{array}{l}\text { Team members develop concrete plans for improving the } \\
\text { selected activities using a series of prompts based on the } \\
\text { 'five whys' principle [36]. This aims to uncover the root causes } \\
\text { of teams'ability or inability to carry out the activities which } \\
\text { influence service user experiences which can act as a precursor } \\
\text { to developing solutions. Guidance to help team members } \\
\text { think creatively and positively about possible solutions is also } \\
\text { included to counteract the tendency for teams to focus on } \\
\text { what they are unable to do. At the end of this phase, teams } \\
\text { complete a service improvement action plan which includes } \\
\text { their planned activities, who is responsible for the activity, and } \\
\text { the expected completion date }\end{array}$ & $\begin{array}{l}\text { Why doesn't this activity happen? Why don't we do it? } \\
\text { What can we do to address this? } \\
\text { How can we check that this activity is important to our } \\
\text { service users? } \\
\text { How can we get feedback from our service users about } \\
\text { our improvement plans? } \\
\text { How can we monitor the success of our planned activi- } \\
\text { ties? }\end{array}$ \\
\hline
\end{tabular}

\section{Strengths and limitations}

- The main strengths of this study are its collaborative design, its focus on developing a practical tool with real-world application and its focus on service user experience.
- The main limitations of this study are its focus on one geographical area in the United Kingdom and limited data on how the framework was implemented over time. 


\section{Interview schedule}
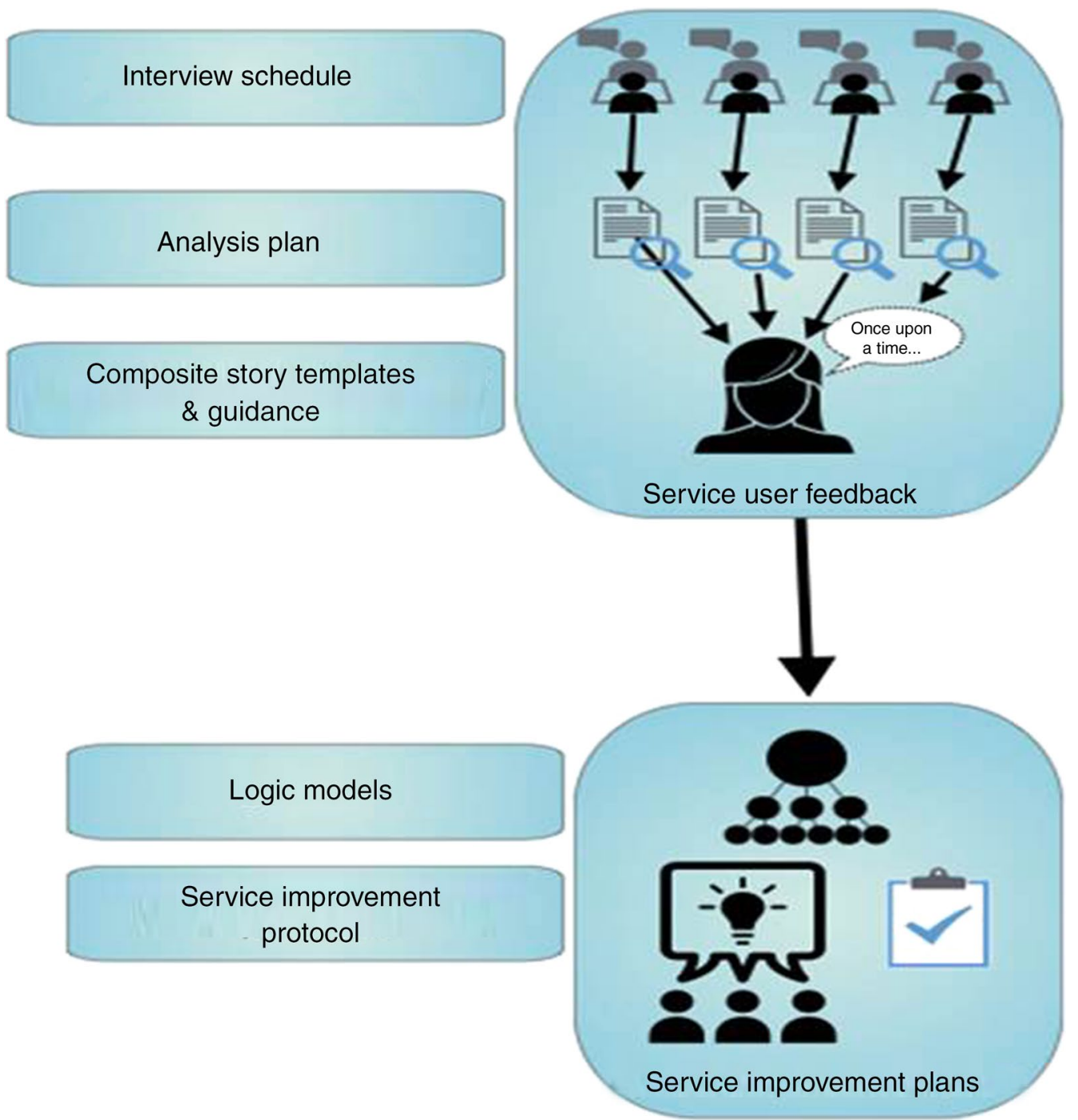

Fig. 2 Using the SUFFICE materials in practice

\section{Additional files}

Additional file 1: Appendix S1. SUFFICE logic models.

Additional file 2: Appendix S2. SUFFICE service user interview schedule.

Additional file 3: Appendix S3. SUFFICE analysis template.

Additional file 4: Appendix S4. SUFFICE service improvement protocol.

\section{Abbreviations}

SUFFICE: Service User Feedback Framework for Improving integrated CarE; NHS: National Health Service; UK: United Kingdom; GP: general practitioner.

\section{Authors' contributions}

VW led the conception and design of the project, reviewed the teamworking literature and led the development of the logic model and service improvement protocol. She has also taken responsibility for drafting and finalising the paper. LP reviewed the service user and integrated care literature, designed, implemented and analysed the interviews with managers and contributed to the service user interview and analysis plan and the service improvement protocol. She contributed to the first draft of this paper and has been involved in revising this version for publication. GF contributed to the service user interview and analysis plan and the service improvement protocol and supported our partner organisations to implement the framework. He has been involved in drafting and revising this paper for publication. All authors read and approved the final manuscript.

\section{Acknowledgements}

The authors would like to acknowledge members of our project advisory group (Emily Brown, Rachel Cooper, Susanne Cox, Allan House, Mussarat Khan, Hailey Matheson, Paul Morrin, Rhona Neilson) and their respective organisations (Leeds Community Healthcare NHS Trust, Leeds Older People's Forum, West Yorkshire \& Bassetlaw Commissioning Support Unit, Leeds Institute of Health Sciences and Leeds City Council). We would also like to thank the other representatives of our partner organisations who participated in interviews and workshops to help us to develop the SUFFICE framework. 


\section{Competing interests}

The authors declare that they have no competing interests.

\section{Data sharing statement}

No clinical data was collected during this project, and no unpublished data are available to other parties.

\section{Disclaimer}

This work was part-funded under the National Institute for Health Research Collaborations for Leadership in Applied Health Research and Care (NIHR CLAHRC) for LYB (Leeds, York and Bradford) — a collaboration between two universities, the NHS, and Social Services. The views and opinions expressed in this paper are those of the authors and not necessarily those of the NHS, the $\mathrm{NIHR}$, or the Department of Health.

\section{Ethics approval}

Ethical approval for the development of the framework was granted by the University of Leeds Medicine \& Health Ethics Committee (SoMREC/13/015). Written consent was given by all participants.

\section{Funding}

This project was supported by the National Institute of Health Research (KRD/012/001/006) and by the Leeds South and East Clinical Commissioning group.

Received: 13 January 2016 Accepted: 18 August 2016

Published online: 08 September 2016

\section{References}

1. Department of Health. Caring for our future: reforming care and support. London: Department of Health; 2012.

2. Naylor C, Imison C, Addicott R, et al. Transforming our health care system: ten priorities for commissioners. London: The Kings Fund; 2013.

3. National Voices. 9 big shouts: patients and service users respond to the health reforms. London: National Voices; 2011.

4. NHS Future Forum. Integration: a report from the NHS future forum. London: NHS Future Forum; 2012

5. National Collaboration for Integrated Care and Support. Integrated care and support: our shared commitment. London: National Collaboration for Integrated Care and Support; 2013.

6. Goodwin N, Smith J, Davies A, et al. Integrated care for patients and populations: improving outcomes by working together. London: The Kings Fund; 2012.

7. RAND Europe. National evaluation of the Department of Health's integrated care pilots. London: RAND Europe; 2012.

8. https://www.gov.uk/government/news/integration-pioneers-leadingthe-way-for-health-and-care-reform-2. Accessed Jan 2015

9. Harris M, Greaves F, Gunn L, et al. Multidisciplinary group performancemeasuring integration intensity in the context of the north west London integrated care pilot. Int J Integr Care. 2013;13:e041.

10. Roland M, Lewis R, Steventon A, et al. Case management for at-risk elderly patients in the English integrated care pilots: observational study of staff and patient experience and secondary care utilisation. Int J Integr Care. 2012;12:e130

11. Ham C. Working together for health: achievements and challenges in the Kaiser NHS beacon sites programme. Birmingham: Health Services Management Centre; 2010.

12. Curry N, Ham C. Clinical and service integration: the route to improved outcomes. London: The Kings Fund; 2010.

13. Wilberforce M, Harrington V, Brand C, Tucker S, Abendstern M, Challis D. Towards integrated community mental health teams for older people in England: progress and new insights. Int J Geriatr Psychiatry. 2011;26(3):221-8.

14. Maslin-Prothero SE, Bennion AE. Integrated team working: a literature review. Int J Integr Care. 2010;10:e043.
15. Brown L, Domokos T, Tucker C. Evaluating the impact of integrated health and social care teams on older people living in the community. Health Soc Care Community. 2003;11(2):85-94.

16. Ling T, Brereton L, Conklin A, Newbould J, Roland M. Barriers and facilitators to integrating care: experiences from the English integrated care pilots. Int J Integr Care. 2012;12:e129.

17. Belling $\mathrm{R}$, Whittock $M$, McLaren $S$, et al. Achieving continuity of care: facilitators and barriers in community mental health teams. Implement Sci. 2011:6:23 doi:10.1186/1748-5908-6-23.

18. Greenfield G, Ignatowicz AM, Belsi A, Pappas Y, Car J, Majeed A, Harris M. Wake up, wake up! It's me! It's my life! patient narratives on person-centeredness in the integrated care context: a qualitative study. BMC Health Serv Res. 2014;14:619.

19. Graham C, Killpack C, Raleigh V, Redding D, Thorlby R, Walsh J. Options appraisal on the measurement of people's experiences of integrated care. London: Picker Institute Europe; 2013.

20. Hills M, Mullett J, Carroll S. Community-based participatory action research: transforming multidisciplinary practice in primary health care. Pan Am J Public Health. 2007;21(2-3):125-35.

21. Harris R, Sims S, Hewitt G, et al. Interprofessional teamwork across stroke care pathways: outcomes and patient and carer experience. Southampton: NIHR Service Delivery and Organisation programme; 2013.

22. Cameron A, Macdonald G, Turner W, Lloyd L. The challenges of joint working: lessons from the supporting people health pilot evaluation. Int J Integr Care. 2007;7:e39

23. Patton MQ. Developmental evaluation: applying complexity concepts to enhance innovation and use. New York: The Guildford Press; 2011.

24. Glasby J, Dickinson H, Nicoll T, Fleischmann P. Integrated adult health and social care teams in Leeds: a baseline study to inform future evaluation. London: Social Care Institute for Excellence; 2012.

25. National Voices. A narrative for person-centred coordinated care. http:// www.england.nhs.uk/wp-content/uploads/2013/05/nv-narrative-cc.pdf. Accessed Dec 2013.

26. Malley J, Caiels J, Fox D, et al. A report on the development studies for the National Adult Social Care User Experience Survey. Canterbury: Personal Social Services Research Unit; 2010.

27. Bryman A. Social research methods. 4th ed. Oxford: Oxford University Press; 2012

28. Gillard S, Simons L, Turner K, Lucock M, Edwards C. Patient and public involvement in the coproduction of knowledge: reflection on the analysis of qualitative data in a mental health study. Qual Health Res. 2010;22(8):1126-37.

29. Allam S, Blyth S, Fraser A, et al. Our experience of collaborative research: service users, carers and researchers work together to evaluate an assertive outreach service. J Psychiatr Ment Health Nurs. 2004;11(3):368-73.

30. Sims S, Brearley S, Hewitt G, Harris R. Involving service users and carers in the analysis of research data. In: RCN annual international nursing research conference. Harrogate: Royal College of Nursing; 2011.

31. Halcomb EJ, Davidson PM. Is verbatim transcription of interview data always necessary? Appl Nurs Res. 2006;19:38-42

32. DeCuir-Gunby J, Marshall P, McCulloch A. Developing and using a codebook for the analysis of interview data: an example from a professional development research project. Field Methods. 2010;23:136.

33. NHS Institute for Innovation and Improvement. Quality and service improvement tools. Secondary quality and service improvement tools. http://www.institute.nhs.uk/quality_and_service_improvement_tools/. Accessed Mar 2013

34. Wilcock PM, Brown GCS, Bateson J, Carver J, Machin S. Using patient stories to inspire quality improvement within the NHS modernization agency collaborative programmes. J Clin Nurs. 2003;12(3):422-30.

35. Thomas H. On patient stories. Health Serv J. 2006;116(6020):23.

36. NHS Institute for Innovation and Improvement. Root cause analysis using five whys. Secondary root cause analysis using five whys. http://www. institute.nhs.uk/quality and service improvement tools/quality and service_improvement_tools/identifying_problems_-_root_cause_analysis_using5 whys.html. Accessed Mar 2013. 